\title{
EFEITO DA PROFUNDIDADE DE TRABALHO NA QUALIDADE DA OPERAÇÃO DE SUBSOLAGEM PARA IMPLANTAÇÃO FLORESTAL ${ }^{1}$
}

\author{
Saulo Boldrini Gonçalves², Eduardo da Silva Lopes ${ }^{3 *}$, Nilton César Fiedler ${ }^{4}$, Karina Maria Vieira Cavalieri ${ }^{5}$, \\ James Stahl ${ }^{6}$ e Carlos Henrique Fonseca Drinko ${ }^{7}$

\footnotetext{
${ }^{1}$ Recebido em 12.12.2014 aceito para publicação em 01.12.2015.

${ }^{2}$ Universidade Estadual do Centro-Oeste, UNICENTRO, Mestrado em Ciências Florestais, Irati, PR - Brasil. E-mail: <sauloboldrinig@hotmail.com>.

${ }^{3}$ Universidade Estadual do Centro-Oeste, UNICENTRO, Departamento de Engenharia Florestal, Irati, PR - Brasil. E-mail: <eslopes@pq.cnpq.br>.

${ }^{4}$ Universidade Federal do Espírito Santo, UFES, Departamento de Ciências Florestais e da Madeira, Jerônimo Monteiro, ES - Brasil. E-mail: <fiedler@pq.cnpq.br>.

${ }^{5}$ Universidade Federal do Paraná,UFPR, Departamento de Solos e Engenharia Agrícola, Curitiba,PR-Brasil.E-mail: karina.cavalieri@ufpr.br

${ }^{6}$ Klabin S.A., Telêmaco Borba, PR - Brasil. E-mail: <jstahl@klabin.com.br>.

${ }^{7}$ Universidade Estadual do Centro-Oeste, UNICENTRO, Graduando em Engenharia Florestal, Irati, PR - Brasil. E-mail: $<$ cdrinko@hotmail.com>.

*Autor para correspondência.
}

\begin{abstract}
RESUMO - Objetivou-se, com este trabalho, realizar uma avaliação da profundidade de trabalho na qualidade da operação de subsolagem para implantação de povoamentos florestais. O experimento foi realizado em uma empresa florestal localizada no Município de Telêmaco Borba, Estado do Paraná, Brasil, em um Latossolo de textura média. O equipamento utilizado foi um trator de esteiras com potência de $170 \mathrm{HP}$ e peso operacional de $19.000 \mathrm{~kg}$, equipado com lâmina tipo V-shear, tracionando um subsolador de arrasto mono-haste com peso de $5.390 \mathrm{~kg}$, com a operação de subsolagem nas profundidades de 40 e $50 \mathrm{~cm}$. A qualidade da subsolagem foi determinada com base nos aspectos de profundidade, formato e distância entre as linhas de sulcos, com uso de gráficos de controle. Os resultados indicaram a falta de controle no processo relacionado com a profundidade e formato das linhas de preparo do solo na operação de subsolagem em ambos os tratamentos. O preparo do solo realizado a $50 \mathrm{~cm}$ de profundidade apresentou os melhores padrões de qualidade nas condições estudadas.
\end{abstract}

Palavras-chave: Subsolagem; Controle de qualidade; Operações florestais.

\section{EFFECT OF DEPTH OF WORK IN THE QUALITY THE SUBSOILING OPERATION FOR FOREST PLANTING}

\begin{abstract}
The objective of this research was to evaluate the effect of work depth in the quality the subsoiling operation for forest planting. The study was conducted in a forest company located in Telemaco Borba, Parana State, Brazil, in an Oxisol of medium texture. The equipment used was a track tractor with power of 170 $H P$ and operational weight of 19,000 kg, equipped with $V$-shear blade, pulling a drag subsoiler mono haste with weigh of $5,390 \mathrm{~kg}$, working at subsoiling operation in the depths of 40 and $50 \mathrm{~cm}$. The quality of the subsoiling was determined based on the aspects of depth, format and distance between the lines to be planted, with the use of control graphics. The results showed the lack of control in process related to the depth and format of the lines of soil preparation in subsoiling operation in both the treatments. The soil preparation carried out at $50 \mathrm{~cm}$ depth showed the best quality standards at the studied conditions.
\end{abstract}

Keywords: Subsoiling; Quality control; Forestry operations. 


\section{INTRODUÇÃO}

A obtenção de elevada produção de madeira depende diretamente da etapa de implantação do povoamento florestal, que por sua vez é influenciado pela espécie, qualidade das mudas, tipo e qualidade do preparo do solo e dos tratos culturais e silviculturais.

O preparo do solo para a implantação de povoamentos florestais visa disponibilizar quantidades suficientes de água e nutrientes para o rápido estabelecimento das mudas e o crescimento do sistema radicular, bem como a eliminação de plantas indesejáveis, evitando a competição (GATTO et al., 2003). Além disso, visa à desagregação de camadas adensadas ou compactadas do solo, diminuindo a resistência do solo à penetração e facilitando o desenvolvimento do sistema radicular das árvores, de modo que possam explorar maior volume de solo com o aumento da absorção de água e nutrientes (LETEY, 1985; FINGER et al., 1996; FERNANDES; SOUZA, 2001).

Atualmente, a tecnologia mais utilizada no preparo do solo em plantios florestais é o cultivo mínimo, que acarreta baixa mobilização mecânica e a manutenção dos resíduos vegetais sobre o solo. Entre as diversas opções de preparo do solo, destaca-se a subsolagem devido aos seus efeitos benéficos ao solo e suas vantagens operacionais e econômicas (GAVA, 2002; SASAKI; GONÇALVES, 2005; RAPER; BERGTOLD, 2007; BLAZIER; DUNN, 2008). Entretanto, apesar de a subsolagem ser uma operação comum e muito empregada nas operações de preparo do solo na maioria das empresas florestais, deve-se ter cautela no seu uso, pois segundo Hillel (1980), em muitos casos, essa operação demanda grande consumo de energia, tempo de execução e elevados custos operacionais e de produção, dependendo diretamente da profundidade de trabalho, do número de operações realizadas, do tipo e umidade do solo, da declividade do terreno etc. Por isso, torna-se necessário o desenvolvimento de técnicas que possibilitem a identificação e o reconhecimento das condições do solo e dos equipamentos na execução das operações, bem como eficiente controle de qualidade do preparo do solo, de modo a garantir o adequado crescimento das plantas com elevada produtividade e redução dos custos da produção florestal.

Segundo Gitlow (1993), a qualidade é o julgamento feito pelos clientes ou usuários de um produto ou serviço. Montgomery (1997) relatou que é a redução da

Revista Árvore, Viçosa-MG, v.40, n.1, p.29-37, 2016 variabilidade de um produto ou serviço e, quanto menor essa variabilidade, melhor a confiabilidade e a aceitação do produto ou serviço pelos clientes, podendo ainda ser entendida como os procedimentos para evitar o desperdício de recursos financeiros, tempo e energia. Já Campos (2004) afirmou que o controle de qualidade é um modelo gerencial centrado no controle do processo, tendo como meta a satisfação das necessidades das pessoas, enquanto Rezende et al. (2000) afirmaram que o controle de qualidade não pode ser voltado apenas para o produto final obtido, devendo abranger todo o processo que envolve as atividades florestais.

Entre os aspectos do controle de qualidade que devem ser considerados nas operações de preparo do solo para implantação florestal, cita-se a profundidade de trabalho, o formato do sulco e a desagregação das camadas compactadas do solo, e tais indicadores criam uma base racional para uma segura ação gerencial (MAIN, 1994).

Taylor e Beltrame (1980) consideraram que a subsolagem pode atingir profundidades de até $80 \mathrm{~cm}$, podendo em solos coesos chegar até 1,10 m (STAPE et al., 2002), enquanto Dalbem et al. (2008) recomendaram a profundidade mínima de $50 \mathrm{~cm}$ no preparo mecanizado de solo para plantio de eucalipto.

Pereira et al. (2012), estudando um subsoladoradubador de arraste de haste única nas operações de preparo mecanizado de solo para o plantio florestal, demonstraram que a declividade de até $40 \%$ permite atingir uma profundidade mínima de $50 \mathrm{~cm}$ na realização de uma operação com qualidade. Gava (2003), quando avaliou a qualidade do preparo do solo em três classes de textura do solo para implantação de eucalipto no Estado de São Paulo com uso de um subsolador tipo mono-haste, verificou que o solo de textura argilosa apresentou restrições à atuação do subsolador, devendo nessas condições receber um preparo específico, enquanto em solo de textura arenosa a qualidade não foi atendida em termos de largura do sulco, por tratar-se de um solo muito friável.

Por sua vez, Lopes et al. (1995), ao aplicarem o controle da qualidade em operações mecanizadas na cultura de cana-de-açúcar, concluíram que os índices de variação do espaçamento entre sulcos nas áreas analisadas foram muito elevados, estando fora de controle, apesar de a média ter ficado próxima da desejada para a operação, sugerindo problemas de má regulagem do implemento ou erros na operação. 
Diante do exposto, este trabalho objetivou avaliar o efeito da profundidade de trabalho na operação de subsolagem para implantação de povoamentos florestais, contribuindo para a melhoria da qualidade, aumento de produtividade e redução dos custos de produção.

\section{MATERIAL E MÉTODOS}

\subsection{Caracterização da área de estudo}

Esta pesquisa foi realizada em uma empresa florestal localizada no Município de Telêmaco Borba, Estado do Paraná, Brasil, nas coordenadas geográficas $24^{\circ} 21^{\prime} 24^{\prime \prime} \mathrm{S}$ e $50^{\circ} 37^{\prime} 18^{\prime \prime} \mathrm{W}$, com altitude média de $760 \mathrm{~m}$. A coleta dos dados foi realizada nos meses de junho e agosto de 2013.

O povoamento existente na área de estudo anteriormente ao preparo do solo era constituído por Pinus taeda, com idade de corte de 32 anos, área basal de 44,3 $\mathrm{m}^{2} \mathrm{ha}^{-1}$, espaçamento de 2,5 x 1,7 m, DAP médio de 41,3 cm e volume médio por hectare de $667 \mathrm{~m}^{3} \mathrm{ha}^{-1}$. O solo foi classificado como Latossolo Vermelho distrófico típico (LVd), de textura muito argilosa e apresentando quantidade média de $644 \mathrm{~g} \mathrm{~kg}^{-1}$ de argila, $159 \mathrm{~g} \mathrm{~kg}^{-1}$ de silte e $197 \mathrm{~g} \mathrm{~kg}^{-1}$ de areia total, com valores de densidade do solo (Ds) entre 1,33 e $1,43 \mathrm{Mg} \mathrm{m}^{-3}$ nas camadas de 0 a $60 \mathrm{~cm}$, enquanto o relevo foi classificado como suave a ondulado.

O sistema de colheita da madeira utilizado na área de estudo foi de árvores inteiras (full tree), sendo a derrubada e empilhamento das árvores realizados por um trator florestal feller-buncher, seguido do arraste das árvores pelo trator florestal skidder com tração $6 \times 6$ e processamento da madeira na margem do talhão pelo harvester.

\subsection{Descrição da operação de subsolagem}

A operação de subsolagem foi realizada por um trator de esteiras com potência bruta de $170 \mathrm{HP}$, rodados de esteiras e peso operacional de $19.000 \mathrm{~kg}$, equipado com uma lâmina tipo $\mathrm{V}$-shear com 3,86 $\mathrm{m}$ de comprimento e $1,16 \mathrm{~m}$ de altura. $\mathrm{O}$ trator tracionou um subsolador de arrasto mono-haste com peso de $5.390 \mathrm{~kg}$, haste em formato reto e ponteira com asas, discos de curva, rolo compressor e reservatório de fertilizantes de 700 $\mathrm{kg}$, regulado para realizar a subsolagem nas profundidades estudadas.

\subsection{Procedimento Amostral}

A avaliação do sistema de preparo do solo foi conduzida no delineamento de blocos casualizados, sendo definidos dois tratamentos: $\mathrm{T} 1=$ profundidade de subsolagem de $40 \mathrm{~cm}(\mathrm{~S}-40)$; e T2 = profundidade de subsolagem de $50 \mathrm{~cm}$ (S-50). Ambos os tratamentos foram aplicados na mesma área de preparo do solo, subdivididos em duas partes iguais com condições homogêneas, sendo as profundidades definidas no ponto central do sulco.

A escolha dos tratamentos S-40 e S-50 ocorreu em acordo com a empresa, pois normalmente a operação de subsolagem era realizada na profundidade de 50 $\mathrm{cm}$ em todas as texturas de solo, devido à praticidade operacional, enquanto em solos arenosos era realizada na profundidade de $40 \mathrm{~cm}$. Assim, buscou-se avaliar, mesmo em solo de textura argilosa, se a redução de $10 \mathrm{~cm}$ na profundidade de subsolagem contribui para o aumento efetivo da profundidade do sulco e a qualidade da operação de subsolagem.

O procedimento de amostragem adotado neste experimento foi uma adaptação de Peche-Filho et al. (1994) e Fernandes (2000), que consideraram cada linha de preparo como uma unidade amostral composta. Foram amostradas 15 linhas de preparo do solo por tratamento, distribuídos na área de forma sistemática, em que em cada linha foram obtidos os dados em 10 pontos de medição, formando uma malha de 150 pontos amostrais por tratamento. As parcelas foram instaladas a uma distância mínima de $20 \mathrm{~m}$ dos carreadores, de forma a permitir a estabilização do equipamento na execução da operação.

\subsection{Coleta de dados}

A qualidade da subsolagem foi avaliada com base nos itens: profundidade e formato dos sulcos e distâncias entre linhas de sulcos. Para cada item foram estabelecidos parâmetros (Tabela 1), determinados a partir de experiências realizadas pela empresa, variando de acordo com as características de textura do solo, espécie e finalidade do plantio.

\subsubsection{Profundidade e formato do sulco}

A profundidade e formato do sulco foram avaliados depois de realizada a subsolagem com uso de uma haste de ferro graduada e um gabarito, confeccionados com adaptações a partir do modelo proposto por Trindade (1993), ilustrado na Figura 1.

Revista Árvore, Viçosa-MG, v.40, n.1, p.29-37, 2016 
Tabela 1 - Parâmetros adotados pela empresa na avaliação da qualidade do preparo do solo.

Table 1 - Parameters adopted by the company at evaluation of soil preparation quality.

\begin{tabular}{lccc}
\hline Item & \multicolumn{2}{c}{ Parâmetro Adotado } \\
\cline { 2 - 4 } & Mínimo & Ideal & Máximo \\
\hline Profundidade do centro do sulco(cm) & 30 & 40 & 50 \\
Distância entre linhas de sulco(m) & 3,8 & 4,0 & 4,2 \\
\hline
\end{tabular}

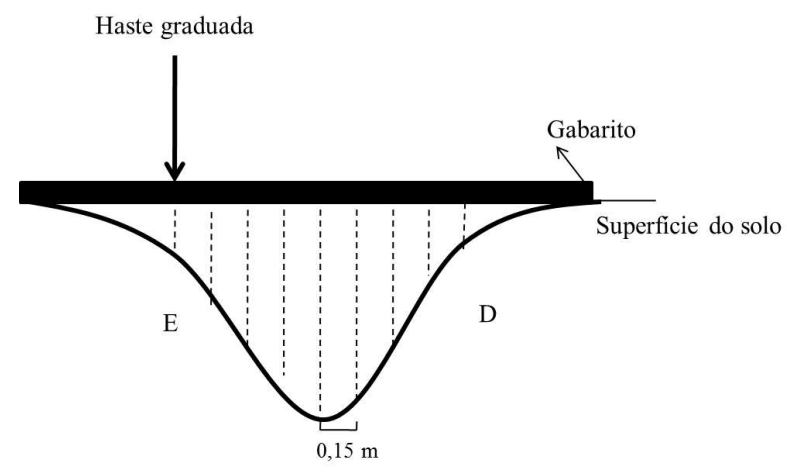

Figura 1 - Esquema da amostragem do formato e profundidade do sulco após o preparo do solo (locações das nove medidas. $\mathrm{E}=$ a esquerda do centro do sulco; e $\mathrm{D}=$ a direita do centro do sulco).

Figure 1 - Scheme of sampling of the shape and depth of the line after tillage (locations of the nine measures. $E=$ left of the center of the line, and $D=$ right of the center of the line).

Em cada uma das linhas de preparo do solo amostradas foram obtidos 10 pontos de medição, distribuídos de forma a abranger as variações ao longo da linha de sulco, sendo, então, realizadas em cada ponto amostral nove medições equidistantes $15 \mathrm{~cm}$ entre si, no sentido da largura do sulco.

As medidas de formato e profundidade foram obtidas de uma extremidade a outra do sulco, sendo os resultados contrastados com as medidas estipuladas pela empresa e verificado se o preparo nos tratamentos propostos estava adequado ao padrão de qualidade desejado.

\subsubsection{Distâncias entre linhas de sulco}

As distâncias entre as linhas de sulco foram avaliadas nos 10 pontos de medição nas linhas de preparo do solo, realizado no sentido perpendicular às linhas e iniciado no primeiro ponto amostral da linha 1 até o primeiro ponto da linha 15 . Os pontos foram medidos a partir do ponto central do sulco de subsolagem ao ponto central da linha adjacente.

Revista Árvore, Viçosa-MG, v.40, n.1, p.29-37, 2016

\subsection{Avaliação da qualidade}

A avaliação da qualidade foi realizada com uso de Gráficos de Controle, seguindo as etapas propostas por Alves e Pieske (1997). O fator de ajuste da distribuição normal $(\mathrm{c} 2=0,922)$ para a estimativa dos limites de controle foram reproduzidos a partir dos estudos de Paladini (1990).

Para a obtenção da estimativa das linhas médias e os limites dos Gráficos de Controle foram utilizadas as equações propostas por Trindade et al. (2000):

$$
\begin{aligned}
& \mathrm{LM}=\mathrm{X} \\
& \mathrm{LSC}=\mathrm{X}+3 \frac{\mathrm{S}}{\mathrm{c} 2 \sqrt{\mathrm{n}}} \\
& \mathrm{LIC}=\mathrm{X}+3 \frac{\mathrm{S}}{\mathrm{c} 2 \sqrt{\mathrm{n}}}
\end{aligned}
$$

Em que LM é a linha média; Xé o valor médio; LSC é o limite superior de controle; Sé o desvio médio; C2 é o fator de ajuste à distribuição normal, tabelado em função de n; né o tamanho da amostra; e LIC é o limite inferior de controle.

\subsection{Análise estatística}

Os valores médios de produtividade do conjuntosubsolador e dos parâmetros de qualidade obtidos nos diferentes tratamentos foram submetidos à análise de variância, sendo, quando significativas, as médias comparadas pelo teste $\mathrm{T}$ a $5 \%$ de probabilidade.

\section{RESULTADOS}

\subsection{Profundidade e formato do sulco}

Na Figura 2 são apresentados os gráficos de controle referente aos valores médios de profundidade e formato do sulco após a realização da subsolagem nos tratamentos $\mathrm{S}-40$ e S-50. Como pode ser visto, os maiores valores de profundidade e formato do sulco em ambos os 

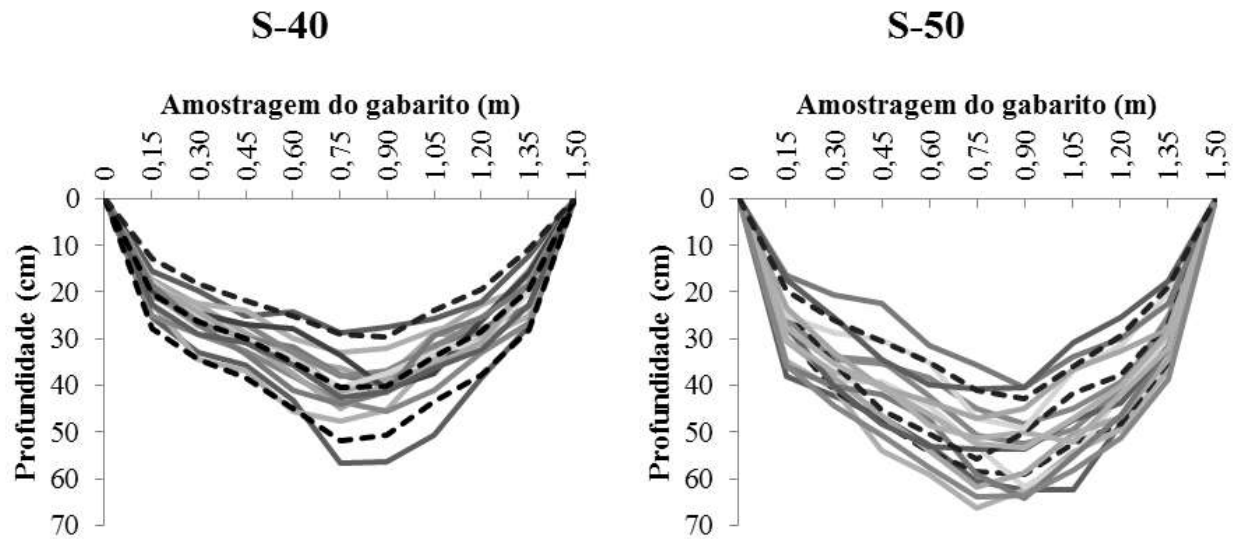

Figura 2 - Gráficos de controle da média da profundidade do preparo do solo dos tratamentos S-40 e S-50. As linhas tracejadas são: Limite Inferior de Controle; Linha média; e Limite Superior de Controle, respectivamente.

Figure 2 - Control graphs for the average depth of tillage of treatments $S-40$ and $S-50$. The dashed lines are: Lower Limit of Control; Midline; and High Limit of Control, respectively.

tratamentos foram observados nas faixas de 60 a 90 $\mathrm{cm}$ e de 60 a $105 \mathrm{~cm}$ do transecto, respectivamente. Tais resultados apresentaram grande variação nos valores de profundidade e formato de sulco ao longo das linhas avaliadas, conferindo maior variação na profundidade de trabalho efetivo do subsolador. Em ambos os tratamentos, verificou-se a existência de pontos fora do limite de controle inferior e superior, situação que caracterizou a falta de um controle eficiente para a variável analisada.

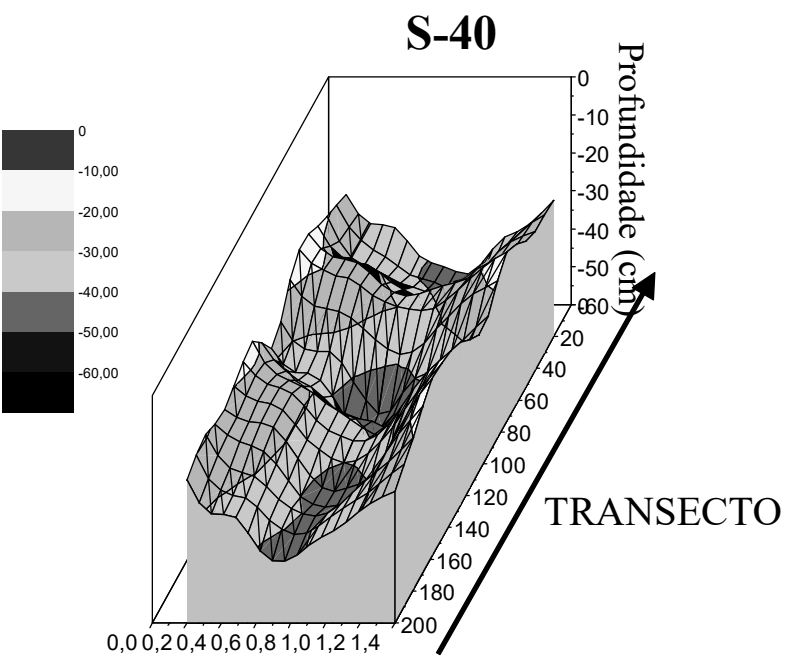

Os resultados médios referentes à profundidade e formato dos sulcos nas parcelas amostrais são ilustrados em três dimensões na Figura 3. É possível observar que o tratamento S-40 apresentou relevo menos íngreme ao longo do transecto, explicado pelo fato de a profundidade e formato do sulco nas linhas subsoladas possuírem menores valores. No tratamento S-50, observou-se maior inclinação no relevo, conferido pela maior profundidade das linhas de sulco ao longo do transecto.

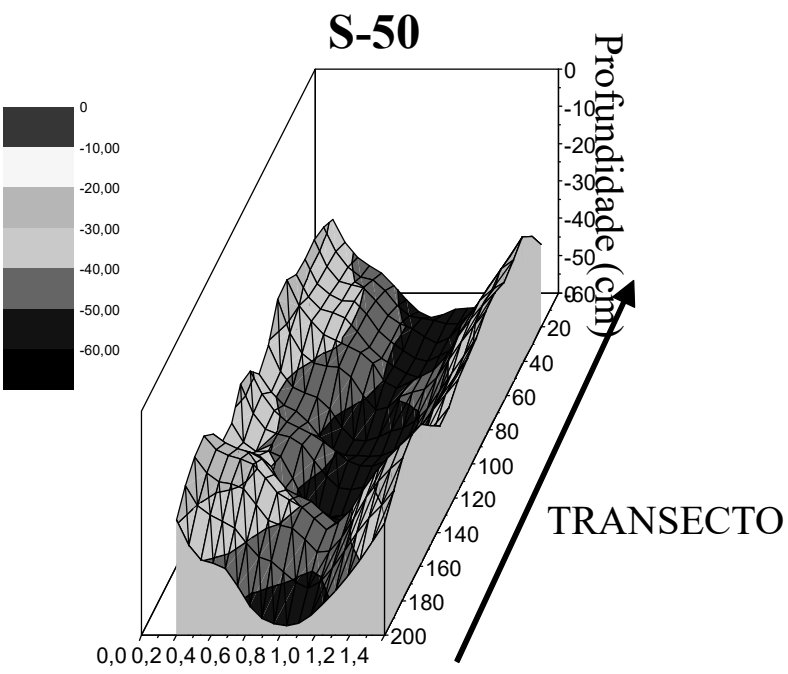

Figura 3 - Variação média do sulco de preparo do solo nas linhas amostrais e nos transectos dos tratamentos S-40 e S50.

Figure 3 - Mean variation of the line of tillage in the sampling lines and transects of treatment S-40 and S-50. 
A profundidade média no centro do sulco em todos os pontos amostrais das linhas de subsolagem no tratamento S-40 foi de $40,5 \mathrm{~cm}$, com variação entre 28,2 e $49,5 \mathrm{~cm}$, enquanto no tratamento S-50 apresentou profundidade média de $52,4 \mathrm{~cm}$, com variação entre 37,1 e $62,8 \mathrm{~cm}$.

\subsection{Distância entre linhas de sulco}

Na Figura 4 são apresentados os histogramas com a distribuição dos valores das distâncias entre as linhas de sulco, em classes para as duas profundidades de subsolagem estudadas. Na subsolagem a $40 \mathrm{~cm}$ de profundidade, a distância média entre as linhas de sulco foi de 4,36 m, com variação de 3,40 a 5,20 m. As classes que incluem a especificação da empresa $(3,80$ a 4,00 e 4,00 a 4,20 m com centro de classe igual a 3,90 e 4,10 $\mathrm{m})$ representaram apenas $24 \%$ dos valores encontrados (Figura 4a). Já na situação de subsolagem a $50 \mathrm{~cm}$ de profundidade a distância média entre as linhas de sulco foi de 4,41 m, com variação de 3,60 a 5,20 m. As classes 3,80 a 4,20 m, que representam as especificações exigidas pela empresa, representaram somente $28,6 \%$ dos valores encontrados (Figura 4b).

A frequência absoluta no tratamento S-40 variou de 8 a 16 entrelinhas amostradas por classe de distância, enquanto no S-50 variou de 1 a 35 entrelinhas por classe de distância entre linhas, perfazendo um total de 150 entrelinhas amostradas, em que 37 ficaram dentro das classes especificadas pela empresa para S-40 e 42 para S-50.

É importante ressaltar que, no tratamento S-40, a frequência relativa do centro das classes de distância entre as linhas variou de 8 a $20 \%$ (centro de classe $<3,50$ e 4,30 m, respectivamente), e seis das nove classes de distância apresentaram valor de $11 \%$ de frequência relativa. Para o S-50, a frequência relativa do centro das classes de distâncias entre linhas variou de 0 a $26 \%$ (classe $<3,50$ e 4,50 m, respectivamente), havendo maior concentração das distâncias entre linhas nas classes próximas aos limites desejados pela empresa, onde as classes de 3,80 a 4,60 englobaram $74 \%$ de todas as medidas amostradas.

\section{DISCUSSÃO}

\subsection{Profundidade e formato do sulco}

Em ambos os tratamentos estudados, os maiores valores de profundidade de sulco foram obtidos nas faixas próximas ao centro do sulco, podendo tais resultados ser explicados pela ação da haste do subsolador que propicia maior profundidade de trabalho nas faixas próximas ao centro do sulco. Sasaki e Gonçalves (2005), ao avaliarem o desempenho operacional de um subsolador mono-haste em função da estrutura, do

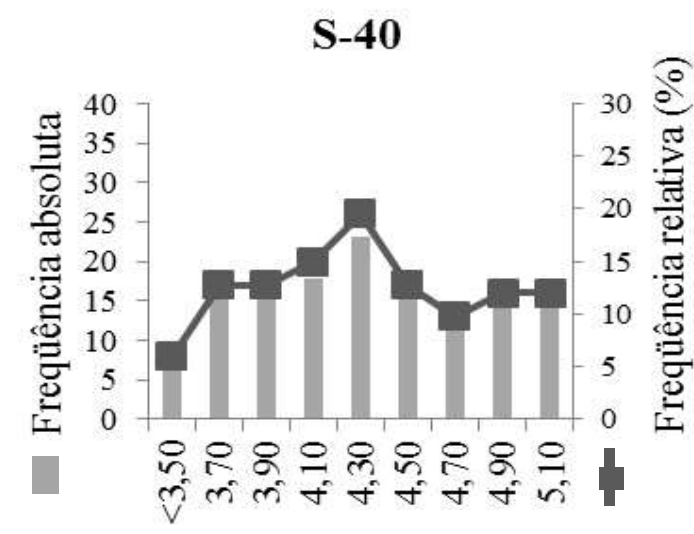

Distância entre linhas (m)

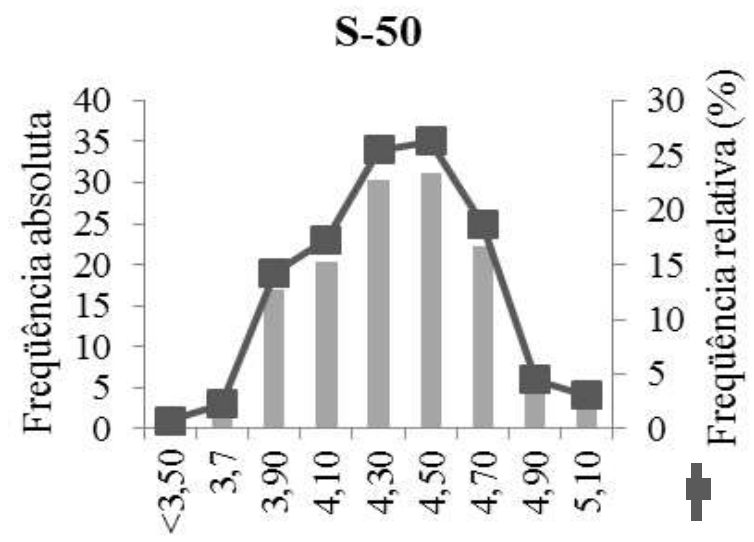

Distância entre linhas (m)

(a)

(b)

Figura 4 - Histograma de distribuição do centro das classes de distância entre linhas de sulco: a) tratamento S-40; b) tratamento S-50 (LE = Linha de Especificação).

Figure 4-Distribution histogram of the center of the distance classes between lines: a) S-40 treatment; and b) S-50 treatment (LE = Line of Specification).

Revista Árvore, Viçosa-MG, v.40, n.1, p.29-37, 2016 
teor de argila e de água em três Latossolos, observaram que os maiores valores de profundidade efetiva de trabalho foram obtidos na faixa de $30 \mathrm{~cm}$ do ponto central do sulco.

A operação de subsolagem foi considerada de grande variabilidade por apresentar ampla variação nos valores de profundidade e formato do sulco em ambos os tratamentos e ao longo das linhas amostrais (Figura 2).

Os pontos fora do limite inferior e superior de controle foram observados em ambos os tratamentos, porém se observa maior quantidade de linhas fora do limite de controle no tratamento S-50, indicando uma falta de controle para a variável analisada. Tal resultado significou que as causas não aleatórias atuaram sobre a variação da profundidade do sulco nas linhas amostradas, em que Trindade et al. (2007) relataram que as variações não aleatórias são aquelas que podem alterar a normalidade do processo. Segundo Bonilla (1994), quando o processo está fora de controle, as causas não são aleatórias e, por isso, se chamam causas especiais, provocando problemas ocasionais, sendo atribuídas aos operadores.

Ao analisar a Figura 3, verifica-se que houve aumento de $23 \%$ nos valores de profundidade média nas linhas de subsolagem no tratamento S-50 comparado ao $\mathrm{S}-40$, cujo resultado pode ser atribuído à maior altura da haste do subsolador nesse tratamento. Além disso, em torno de $55 \%$ dos 150 pontos amostrais no tratamento S-40 apresentaram profundidade no centro do sulco igual ou superior ao limite estabelecido pela empresa $(40 \mathrm{~cm})$ para solos arenosos. Fessel (2003), ao avaliar o preparo mecanizado do solo na profundidade de trabalho da haste de $40 \mathrm{~cm}$, constatou que $16 \%$ dos pontos amostrais das linhas de subsolagem apresentaram profundidade igual ou superior no centro do sulco a $40 \mathrm{~cm}$ (especificação da empresa). Já no tratamento de subsolagem a $50 \mathrm{~cm}$ de profundidade, $84,6 \%$ dos 150 pontos amostrais apresentaram profundidade igual ou superior ao limite estabelecido de $40 \mathrm{~cm}$ (empresa) para o centro do sulco.

O tratamento S-50 apresentou 30\% mais medidas dentro das especificações da empresa em relação ao S-40, podendo tal resultado ser atribuído à diferença de profundidade de trabalho da haste do subsolador de 40 para $50 \mathrm{~cm}$. Assim, o tratamento de subsolagem a $50 \mathrm{~cm}$ atingiu os objetivos da empresa, com $80 \%$ das linhas de subsolagem com profundidade igual ou acima de $40 \mathrm{~cm}$ de profundidade em solos arenosos. Para Sasaki e Gonçalves (2005), a maior profundidade de subsolagem do solo possibilita o aumento da sobrevivência e do crescimento das mudas, proporcionando o alcance das raízes nas maiores profundidades, menor exposição do solo e redução das perdas por erosão.

\subsection{Distância entre linhas de sulco}

Como pode ser observado na Figura 4, 24\% e 28,6\% dos valores da distância entre as linhas de sulco que ficaram dentro das especificações da empresa indicam que ambos os tratamentos não atenderam ao padrão de qualidade exigido pela empresa, e no mínimo $80 \%$ dos valores obtidos entre as linhas de subsolagem deveriam situar-se entre 3,80 e 4,20 m.

É importante ressaltar que, aproximadamente, $72 \%$ das linhas dos tratamentos S-40 e S-50 apresentaram espaçamentos irregulares, que possivelmente prejudicarão as atividades subsequentes ao preparo do solo para implantação e manutenção do povoamento florestal.

No tratamento S-40, houve maior homogeneidade entre as classes de distância entre sulco, em que seis das nove classes avaliadas compreenderam o mesmo percentual de $11 \%$, proporcionando distribuição mais achatada dos valores em relação ao S-50. Para Fessel (2003), deve-se trabalhar para reduzir essa homogeneidade, minimizando-se os valores que excederam os limites especificados pela empresa $(3,80$ a 4,20 m), devendo a média situar-se entre tais limites. Já no tratamento $\mathrm{S}-50$ houve aumento na variação, sendo a frequência de um no centro de classe $<3,50 \mathrm{~m}$ a 35 indivíduos no centro da classe de 4,50 m (Figura 3). Apesar do aumento de variação, houve concentração maior dos dados dentro do padrão desejado, com $74 \%$ da frequência relativa nas classes próximas ao desejado pela empresa (3,80 a 4,60 m), estando em concordância com valores especificados por Fessel (2003).

\section{CONCLUSÃO}

A profundidade de trabalho e o formato do sulco apresentaram dependência em relação à altura da haste do subsolador e, quanto maior a profundidade de subsolagem, melhor o padrão de qualidade obtido na operação do preparo de solo.

Revista Árvore, Viçosa-MG, v.40, n.1, p.29-37, 2016 
As profundidades de trabalho da haste do subsolador não influenciam a qualidade de distâncias entre as linhas de sulcos no momento de realização do preparo do solo.

A subsolagem realizada a $50 \mathrm{~cm}$ de profundidade apresentou os melhores padrões de qualidade em relação aos parâmetros e condição dos solos estudados.

\section{REFERÊNCIAS}

ALVES, N.I.F.; PIESKE, O.R. Controle estatístico de qualidade utilizando o Excel. Piracicaba: ESALQ, Departamento de Matemática e Estatística, 1997. 98p.

BLAZIER, M.A.; DUNN, M. Stock type, subsoiling, and density impact productivity and land value of a droughty site. Southern Journal of Applied Forestry, v.32, n.4, p.154-162, 2008.

BONILLA, J.A. Qualidade total na agricultura: fundamentos e aplicações. 2.ed. Belo Horizonte: Centro de Estudos de Qualidade Total na Agricultura, 1994. 344p.

CAMPOS, V.F. TQC - Controle da Qualidade Total (no estilo japonês). 8.ed. Nova Lima, MG: INDG Tecnologia e Serviços, 2004. 256p.

DALBEM, M.; DAL'COL, J.; SARCINELLI, T. S.; CARMO, A. P. Fomento Legal: Cultivando o eucalipto. Aracruz: Pauta 6, 2008. 50 p.

FESSEL, V.A.G. Qualidade, desempenho operacional e custo de plantios, manual e mecanizado, de Eucalyptus grandis, implantados com cultivo mínimo de solo. 2003. Dissertação (Mestrado em Recursos Florestais) - Escola Superior de Agricultura "Luiz de Queriroz", Universidade de São Paulo, Piracicaba, 2003.

FERNANDES, R.A.T. Aplicação de conceitos da qualidade em operações mecanizadas na produção de milho (Zea mays L.) para silagem. 2000. $81 \mathrm{f}$. Dissertação (Mestrado em Recursos Florestais) Escola Superior de Agricultura "Luiz de Queiroz", Universidade de São Paulo, Piracicaba, 2000.

Revista Árvore, Viçosa-MG, v.40, n.1, p.29-37, 2016
FERNANDES, H.C.; SOUZA, A.P. Compactação de solos florestais: uma questão para estudos. Revista Árvore, v.25, n.3, p.387-392, 2001.

FINGER, C.A.G.; SCHUMACHER, M. V.; SCHNEIDER, P. R.; HOPPE, J. M. Influência da camada de impedimento no solo sobre o crescimento de Eucalyptus grandis (Hill) ex Maiden. Ciência Florestal, v.6, n.1, p.137145,1996 .

GAVA, J.L. Avaliação da qualidade do preparo do solo em áreas de eucalipto plantado no sistema de cultivo mínimo do solo. Boletim de Pesquisa Florestal, n.47, p.55-68, 2003.

GAVA, J.L. Cultivo mínimo de solos com textura arenosa em áreas planas e suave-ondulada. In: GONÇALVES, J.L.M.; STAPE, J.L. Conservação e cultivo de solos para plantações florestais. Piracicaba: IPEF, 2002. p.221-244.

GATTO, A.; NAIRAM, F. B.; NOVAIS, R. F.; COSTA, L. V.; NEVES, J. C. L. Efeito do método de preparo do solo, em área de reforma, nas suas características, na composição mineral e na produtividade de plantações de Eucalyptus grandis. Revista Árvore, v.27, n.5, p.635-646, 2003.

GITLOW, H.S. Planejando a qualidade, a produtividade e a competividade. Rio de Janeiro: Qualitymark, 1993. 182p.

\section{MONTGOMERY, D.C. Introduction to} statistical quality control. 3.ed. New York: J. Wiley, 1997.677p.

HILLEL, D. Applications of soil physics. New York: Academic Press, 1980. 385p.

LETEY, J. Relantion ship between soil physical properties and crop production. Advances in Soil Science, v.1, p.277-294, 1985.

LOPES, M.B.; MILAN, M.; COELHO, J.L.D. Qualidade em operações agrícolas mecanizadas na cultura de cana-de-açúcar. Stab: Açúcar, Álcool e Subprodutos, v.13, n.1, p.26-30, 1995.

MAIN, J. Guerras pela qualidade: os sucessos e os fracassos da revolução da qualidade. Rio de Janeiro: Campus, 1994. 459p. 
PALADINI, E.P. Controle de qualidade: uma abordagem abrangente. São Paulo: Atlas, 1990.239p.

PEREIRA, D.P.; FIEDLER, N. C.; LIMA, J. S. S.; GUIMARÃES, P. P.; MORA, R.; CARMO, F. C. A. Eficiência da subsolagem na profundidade de preparo do solo em função da declividade do terreno. Cerne, v. 18, n.4, p.607- 612, 2012.

PECHE-FILHO, A.; COSTA, J. A.; FERRETI, G.; STORINO, M. Avaliação do grau de picagem de material orgânico: uma proposta de metodologia. In: CONGRESSO BRASILEIRO DE ENGENHARIA AGRÍCOLA, 23., Campinas, 1994. Resumos. Campinas: UNICAMP; SBEA, 1994. P.2525.

RAPER, R.L.; BERGTOLD, J.S. In-row subsoiling: a review and suggestions for reducing cost of this conservation tillage operation. Applied Engineering in Agriculture, v.23, n.4, p.463-471, 2007.

REZENDE, J.L.P.; JACOVINE, L. A. G.; LEITE, H. G.; TRINDADE, C. Avaliação da qualidade na colheita florestal semimecanizada. Scientia Forestalis, n.57, p.13-26, 2000.

SASAKI, C.M.; GONÇALVES, J.L.M. Desempenho operacional de um subsolador em função da estrutura, do teor de argila e de água em três
Latossolos. Scientia Forestalis, n.69, p.115$124,2005$.

STAPE, J.L.; ANDRADE, S.; GOMES, A. N.; KREJCI, L. C.; RIBEIRO, J. A. Definição de métodos de preparo de solo para silvicultura em solos coesos do litoral norte da Bahia. In:

GONÇALVES, J.L.M.; STAPE, J.L. Conservação

e cultivo de solos para plantaçoes

florestais. Piracicaba: IPEF, 2002. p.259-296.

TAYLOR, J.C.; BELTRAME, L.F.S. Por que, quando e como utilizar a subsolagem. Lavoura Arrozeira, v.3, n.1, p.34-44, 1980.

TRINDADE, C. Desenvolvimento de um sistema de controle de qualidade para a atividade florestal. 1993. 16f. Dissertação (Mestrado em Ciência Florestal) - Universidade Federal de Viçosa, Viçosa, MG, 1993.

TRINDADE, C. Análise da gestão da qualidade na empresa florestal. 2000. 141f. Tese (Doutorado em Ciência Florestal) - Universidade Federal de Viçosa, Viçosa, MG, 2000.

TRINDADE, C.; REZENDE, J. L. P.; JACOVINE, L. A. G.; SARTÓRIO, M. L. Ferramentas da qualidade: aplicação na atividade florestal. 2.ed. Viçosa, MG: Universidade Federal de Viçosa, 2007. 159p. 